\title{
Comparison of a second-generation trabecular bypass (iStent inject) to ab interno trabeculectomy (Trabectome) by exact matching
}

\author{
Yousef Al Yousef ${ }^{1} \cdot$ Alicja Strzalkowska ${ }^{1} \cdot$ Jost Hillenkamp ${ }^{1} \cdot$ André Rosentreter $^{2} \cdot$ Nils A. Loewen ${ }^{1}$ (D) \\ Received: 1 December 2019 / Revised: 7 September 2020 / Accepted: 10 September 2020 / Published online: 22 September 2020 \\ (C) The Author(s) 2020
}

\begin{abstract}
Purpose To achieve a highly balanced comparison of trabecular bypass stenting (IS2, iStent inject) with ab interno trabeculectomy ( $\mathrm{T}$, Trabectome) by exact matching.

Methods Fifty-three IS2 eyes were matched to 3446 T eyes. Patients were matched using exact matching by baseline intraocular pressure (IOP), the number of glaucoma medications, and glaucoma type, and using nearest neighbor matching by age. Individuals without a close match were excluded. All surgeries were combined with phacoemulsification.

Results A total of 78 eyes (39 in each group) could be matched as exact pairs with a baseline IOP of $18.3 \pm 5.1 \mathrm{mmHg}$ and glaucoma medications of $2.7 \pm 1.2 \mathrm{in}$ each. IOP in IS2 was reduced to $14.6 \pm 4.2 \mathrm{mmHg}$ at 3 months and in T to a minimum of $13.1 \pm 3.2 \mathrm{mmHg}$ at 1 month. In IS2, IOP began to rise again at 6 months, eventually exceeding baseline. At 24 months, IOP in IS2 was $18.8 \pm 9.0 \mathrm{mmHg}$ and in T $14.2 \pm 3.5 \mathrm{mmHg}$. IS2 had a higher average IOP than T at all postoperative visits $(p<0.05$ at 1, 12, 18 months). Glaucoma medications decreased to $2.0 \pm 1.5$ in IS2 and to $1.5 \pm 1.4$ in T.

Conclusion T resulted in a larger and sustained IOP reduction compared with IS2 where a rebound occurred after 6 months to slightly above preoperative values.
\end{abstract}

\section{Key messages}

- Both trabecular bypass stents and trabecular ablation are microincisional glaucoma surgeries that improve conventional outflow. Implanted stents can suffer from biofilm deposition and fibrosis around six months.

Exact matching of iStent inject to Trabectome patients in this study revealed a loss of effect in iStent patients at six months. The two surgeries had an equally good safety profile.

Keywords Glaucoma surgery $\cdot$ iStent $\cdot$ Trabecular bypass stent $\cdot$ Trabectome $\cdot$ Ab interno trabeculectomy $\cdot$ Exact matching

\section{Introduction}

Following reports of fibrosis [1] and biofilm deposition [2] after trabecular bypass device implantation, we recently used exact

Nils A. Loewen

loewen.nils@gmail.com

1 Department of Ophthalmology, University of Würzburg, Josef-Schneider-Straße, 1197080 Würzburg, Germany

2 Department of Ophthalmology, University Witten/Herdecke, Wuppertal, Germany matching to compare a first-generation trabecular bypass stent (IS1, iStent, Glaukos Corp., San Clemente, CA) to trabecular ablation (T, Trabectome, MicroSurgical Technology, Redmond, WA) [3]. Many surgeons now use microincisional glaucoma surgeries (MIGS) first before considering more extensive, traditional procedures (trabeculectomy, tube shunt) because they have a lower complication rate [4] and shorter procedure time, which allows them to be combined with outpatient cataract surgery. In eyes with stents, we found evidence of declining function in the form of increasing IOP and glaucoma medications above preoperative levels while IOP and medications reduced in matched eyes that had undergone trabecular meshwork (TM) 
ablation [3]. In the present study, we examined whether a smaller, second-generation trabecular bypass stent (IS2, iStent inject, Glaukos Corp., San Clemente, CA) would behave differently. Both the IS1 and the IS2 are made of heparin-coated titanium, but two IS2s are implanted in one session instead of just one IS1. We compared IS2 with T using exact matching, an advanced statistics method developed for a highly balanced comparison $[3,5,6]$. We hypothesized that this method would again reveal significant effect differences between IS2 and T that can be missed in studies that use group averages [7, 8] and may be caused by chronic responses at that the implant site [9-12].

\section{Methods}

\section{Study design}

The study was approved by the local ethics committee of the University of Würzburg (protocol \#20191016 01). Because of its retrospective nature, informed consent was waived. The study was in accordance with the 1964 Helsinki declaration and its later amendments or comparable ethical standards. This study included all patients who underwent either IS2 or $\mathrm{T}$ in our clinic and associated satellites between January 2008 and March 2018. The indication for surgery was a stable IOP with a desire to reduce glaucoma medications at the time of cataract surgery or an above-target IOP as determined by a glaucoma specialist, while on maximally tolerated topical treatment. To increase the chances of an exact match to the new IS2, of which fewer data exist, data from the Trabectome Study Group database $[13,14]$ was used to bring the number of $T$ available for an exact match to 3446 . We excluded patients younger than 20 years of age, with neovascular or uveitic glaucoma, uncontrolled uveitis, or prior ocular surgery. At baseline, we assessed patient history and obtained demographic data, type, and stage of glaucoma; best-corrected visual acuity (BCVA); intraocular pressure (IOP); and the number of glaucoma medications. At each follow-up visit, we recorded BCVA, IOP, and the number of glaucoma medications. The primary outcome was a $\geq 20 \%$ reduction of IOP compared with baseline or an IOP of less than $21 \mathrm{mmHg}$ to better reflect the most common real-world use pattern of the IS2 as an add-on surgery at the time of cataract surgery rather than a principal glaucoma surgery. We combined both IS 2 and $\mathrm{T}$ with phacoemulsification and intraocular lens implantation in all cases. The treating specialists could decide what glaucoma drops to use or whether another glaucoma surgery was needed.

\section{Statistics}

Data were described as frequency, percentage, mean $\pm \mathrm{SD}$, median, and range. Continuous and categorical variables were compared with the Mann-Whitney $U$ test and chi-squared test.
Using exact matching, both groups were matched using preoperative IOP, glaucoma medications, and type of glaucoma, and using Nearest Neighbor Matching for age [15]. Each unit in group 1 (IS2) was matched using exact matching to all possible control units in group $2(\mathrm{~T})$, whereas nearest neighbor matching selected the best matches based on the distance to the value in group 1. $p$ values of less than 0.05 were considered statistically significant. Mean $\pm \mathrm{SD}$ was used to express continuous variables. Statistical analyses were performed using R [16].

\section{Surgical technique}

IS2 and T were combined with phacoemulsification and lens implantation in all cases. IS2 implantation was done after cataract surgery, while $\mathrm{T}$ was done first and followed by cataract surgery. IS2 was implanted through a temporal clear corneal incision and with viscoelastic [17]. Schlemm's canal was identified by allowing blood from the episcleral veins to reflux in relative hypotony. Under direct gonioscopic view, the tip of the inserter was placed against the nasal TM. The stents were inserted by piercing through the TM into Schlemm's canal before releasing. The inserter was retracted, and the viscoelastic removed [17].

$\mathrm{T}$ was performed as described before [18]. Briefly, a 1.6-mm uniplanar, temporal clear corneal incision was created. Under direct gonioscopic visualization, the tip of the handpiece was inserted into Schlemm's canal, and the TM was ablated counterclockwise, followed by clockwise ablation with a total length of around $120^{\circ}$ [19-21]. Ablation was started with the power set to $0.8 \mathrm{~mW}$ and increased as necessary. The handpiece was withdrawn from the anterior chamber. T was done before cataract surgery to provide the highest corneal transparency for angle surgery and because this incision is smaller than the one needed for cataract surgery. After T, a viscoelastic device was injected to form the anterior chamber before enlarging the clear corneal incision for cataract surgery.

In both IS2 and T, postoperative treatment comprised a topical antibiotic for 1 week and a steroid tapered over 4 weeks. Glaucoma medications were stopped on the day of surgery and restarted as needed.

\section{Results}

Of the 53 IS2 eyes, 39 could be matched to T to create 39 nearidentical pairs. Due to exact matching, there was no difference in IOP, the number of IOP-lowering medications, glaucoma type, or VF loss between groups $(p>0.05)$. Table 1 shows the baseline characteristics of each group. IS2 and T had the same preoperative IOP of $18.3 \pm 5.1 \mathrm{mmHg}$. IS2 reached an IOP trough of $14.6 \pm 4.2$ at 3 months ( $p=0.04$ compared with preoperative). IOP continued to slowly increase in IS2 from 6 months onward, eventually reaching the baseline average ( $p>$ 0.05 , Fig. 1a). Thad a minimum IOP of $13.1 \pm 3.2 \mathrm{mmHg}$ at 1 
Table 1 Demographics
$\mathrm{T}, n=39$
IS $2, n=39$

Age

\begin{tabular}{lll} 
Mean \pm SD & $73 \pm 10$ & $72 \pm 8$ \\
Range & $(52,91)$ & $(57,84)$ \\
Gender & & \\
Female & $21(54 \%)$ & $22(56 \%)$ \\
Male & $16(41 \%)$ & $17(44 \%)$ \\
NR & $2(5 \%)$ & $0(0 \%)$ \\
Diagnosis & & \\
POAG & $25(64 \%)$ & $25(64 \%)$ \\
Pseudoexfoliation glaucoma & $11(28 \%)$ & $11(28 \%)$ \\
Angle closure glaucoma & $1(3 \%)$ & $1(3 \%)$ \\
Normal tension glaucoma & $2(5 \%)$ & $2(5 \%)$ \\
Prior surgeries & & $3(8 \%)$ \\
ALT & $1(3 \%)$ & $3(8 \%)$ \\
SLT & $7(18 \%)$ & $0(0 \%)$ \\
Trabeculectomy & $0(0 \%)$ & $39(100 \%)$ \\
Combined surgeries & & \\
Trabectome + phaco & $39(100 \%)$ & \\
\hline
\end{tabular}

month $(p=0.01$, Fig. 1a) and stayed at that level $(p<0.02$ all time points compared with preoperative). At 24 months, IOP in IS2 was $18.8 \pm 9.0 \mathrm{mmHg}$, while in T, it was $14.2 \pm 3.5$ $\mathrm{mmHg}$. T had a lower average IOP than IS2 at all postoperative visits ( $p<0.05$ at $1,12,18$ months, Table 2$)$.

Glaucoma medications started at a matched value of $2.7 \pm$ 1.2 in IS2 and T. In IS2, they decreased to $1.7 \pm 1.4$ by month three $(p=0.04)$, increased to a slightly higher average starting at 6 months, and became similar preoperative counts by month 12 $(p>0.05)$. This medication increase did not prevent the IOP from rising. At 24 months, the medication count in IS2 was 2.0 \pm 1.5 ( $p>0.05$ compared with preoperative, Fig. 1b). In T, medications decreased from the matched count of $2.7 \pm 1.2$ at baseline, became significantly lower at 6 months $(1.6 \pm 1.3, p=$ 0.03 ), and declined to $1.5 \pm 1.4$ at 24 months ( $p=0.04$, Fig. $1 b)$.

Using a definition of success commonly applied to trabecular bypass stents, a final IOP of $\leq 21 \mathrm{mmHg}$ or a $20 \%$ IOP reduction from baseline, $97 \%$ of T and $95 \%$ of IS2 achieved this goal (Fig. 2). One patient in T and two in IS2 required a second surgery. No intra- or postoperative vision-threatening complications such as choroidal effusion, sustained hypotony, choroidal hemorrhage, or infection occurred.

\section{Discussion}

This study applied exact matching to detect essential differences between IS2 and T using real-world data. Studies that use simple statistics or study design can miss those [8, 22]. Exact matching is a non-parametric method of controlling the confounding influence of pretreatment variables [23]. Each patient from one group is matched to a patient from the other group with exactly the same primary values and covariates $[24,25]$. Exact matching is well-suited to compare similar pathological conditions and similar treatments, but a downside is that a certain number of datasets must be excluded from the analysis because the algorithm accepts only identical matches.

IS2 is a second-generation trabecular bypass stent [17] made of the same material as the IS1 (iStent, Glaukos Corp., San Clemente, CA) but smaller [26], allowing to fit two into one injector. The IS2 is implanted using a TM-puncturing forward movement that simplifies the technique and doubles the chance to place the device in Schlemm's canal. In contrast, $\mathrm{T}$ eyes do not retain an implant as the TM is molecularized using plasma $[18,27]$.

In the IS2 patients followed here, we observed a slow loss of efficacy after 6 months. By 24 months, the IOP became indistinguishable from preoperative values and appeared to trend towards exceeding it. In contrast, T had a $20 \%$ reduced IOP through the study. Medications in IS2 were increased around 6 months but not above the preoperative level. In T, medications declined throughout the study. Our experience with the IS2 resembled the IS1 [3] except that IS1 patients had an IOP increase above baseline by $15 \%$ despite counteracting this trend by using $30 \%$ more glaucoma medications compared with the matched $\mathrm{T}$ eyes.
Table 2 Mean IOP and number of medication for Trabectome and iStent groups at each follow-up time point (Welch two-sample $t$ test, significance level set at $\leq$ $0.05)$

\begin{tabular}{|c|c|c|c|c|c|c|}
\hline \multirow[t]{2}{*}{ Time } & \multicolumn{2}{|c|}{$\mathrm{IOP}$ mean $\pm \mathrm{SD}$} & \multirow[t]{2}{*}{$p$ value } & \multicolumn{2}{|c|}{$\mathrm{Rx}$ mean $\pm \mathrm{SD}$} & \multirow[t]{2}{*}{$p$ value } \\
\hline & $\mathrm{T}$ & IS2 & & $\mathrm{T}$ & IS2 & \\
\hline Baseline & $18.3 \pm 5.1$ & $18.3 \pm 5.1$ & & $2.7 \pm 1.2$ & $2.7 \pm 1.2$ & \\
\hline 1 month & $13.1 \pm 3.2$ & $15.5 \pm 4.7$ & $<0.01 *$ & $2.3 \pm 1.6$ & $1.9 \pm 1.5$ & 0.89 \\
\hline 3 months & $13.2 \pm 3.3$ & $14.6 \pm 4.2$ & 0.05 & $2.1 \pm 1.6$ & $1.7 \pm 1.4$ & 0.87 \\
\hline 6 months & $14.0 \pm 3.2$ & $14.7 \pm 2.8$ & 0.17 & $1.8 \pm 1.3$ & $1.8 \pm 1.5$ & 0.51 \\
\hline 12 months & $13.3 \pm 2.5$ & $16.5 \pm 5.2$ & $0.01 *$ & $1.6 \pm 1.3$ & $1.9 \pm 1.5$ & 0.16 \\
\hline 18 months & $14.2 \pm 2.7$ & $17.3 \pm 6.8$ & $0.04 *$ & $1.8 \pm 1.4$ & $2.0 \pm 1.5$ & 0.27 \\
\hline 24 months & $14.2 \pm 3.5$ & $18.8 \pm 9.0$ & 0.10 & $1.5 \pm 1.4$ & $2.0 \pm 1.5$ & 0.11 \\
\hline
\end{tabular}




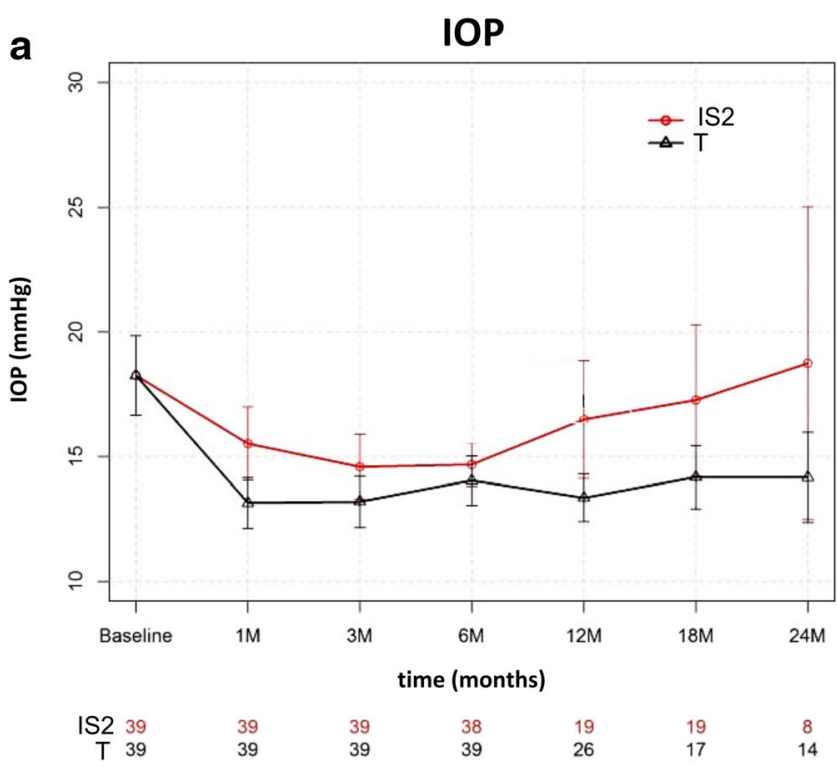

Fig. 1 Mean IOP and number of glaucoma medications for IS2 (iStent inject) and $\mathrm{T}$ (trabectome). a IOP decreased in IS2 through month 3 before starting to rise. In T, IOP decreased through month 1 and remained at this level throughout the study. b Glaucoma medications

IS2 and IS1 are safe add-on procedures that were approved in conjunction with cataract surgery $[17,28]$, and this use pattern continues to be the most common one. In contrast, $\mathrm{T}$ is used across a spectrum of glaucoma severity [29, 30], after failed glaucoma surgery [14, 31], on its own [29], or combined with cataract surgery [30]. The survival rates we computed imply a high clinical success rate for both IS2 and T, but they are based on use criteria of an IOP below $21 \mathrm{mmHg}$ or an IOP reduction by $20 \%$ commonly applied for minimally invasive glaucoma surgery (MIGS). The clinical utility of a medication reduction by 0.7 as achieved here with the IS2 depends on the motivation and goal of the affected individual. It is possible that the progressive loss of effectiveness is related to the fibrosis [1] and biofilm deposition described previously [2]. Although one might expect a more substantial effect from implanting two

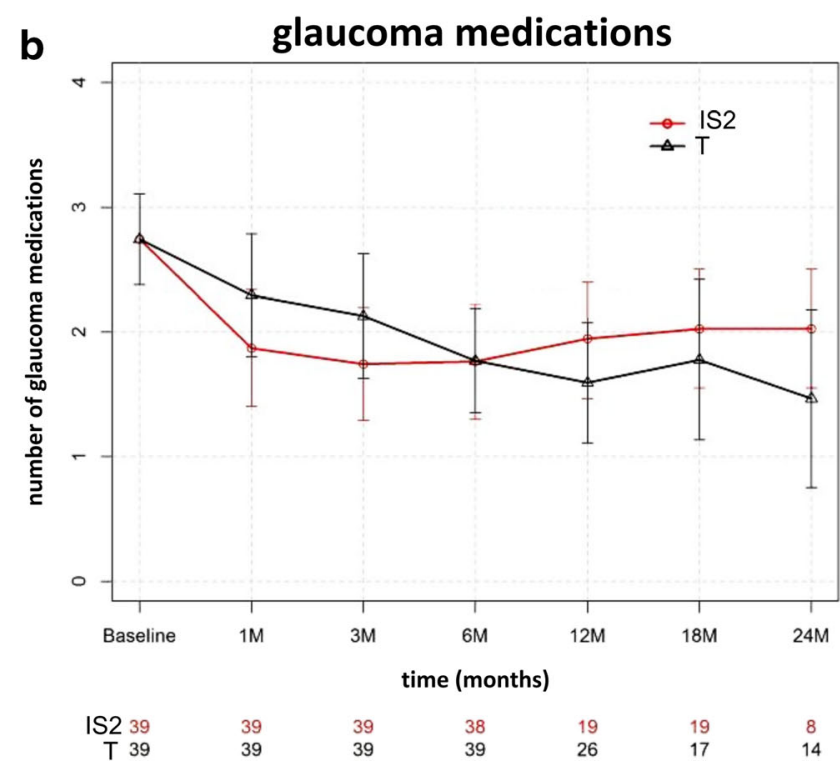

decreased in IS2 before starting to rise again after month 6 . In T, medications decreased throughout the study (mean $\pm \mathrm{SD}$; subject count at each time point)

trabecular bypass IS2 stents than one IS1, the smaller aperture of the IS2 may make it more vulnerable to TM reactivity [9-12].

The lowest IOP any TM bypass or ablation surgery could theoretically achieve is limited by the episcleral venous pressure of about $8 \mathrm{mmHg}$ present in the episcleral venous veins [32]. This limit avoids an excessive pressure reduction that might cause hypotony as can happen after traditional glaucoma surgery [33] and after suprachoroidal [34] or subconjunctival MIGS [35]. It remains unclear why trabecular bypass, disruption, or ablation methods do not routinely achieve a postoperative IOP around $8 \mathrm{mmHg}$. It is possible that post-trabecular resistor elements that have been observed in laboratory studies might play a role $[36,37]$.

Our study had several limitations. It was specifically designed to detect differences between IS2 and T which cannot
Fig. 2 Procedure survival curve for IS2 and T with 24 months of follow-up time indicates a relatively high success rate despite a modest IOP and medication reduction. The criteria were $\mathrm{a} \geq$ $20 \%$ IOP reduction or an IOP of less than $21 \mathrm{mmHg}$ reflecting a common use pattern of IS2 as an add-on surgery at the time of cataract surgery rather than a principal glaucoma surgery survival

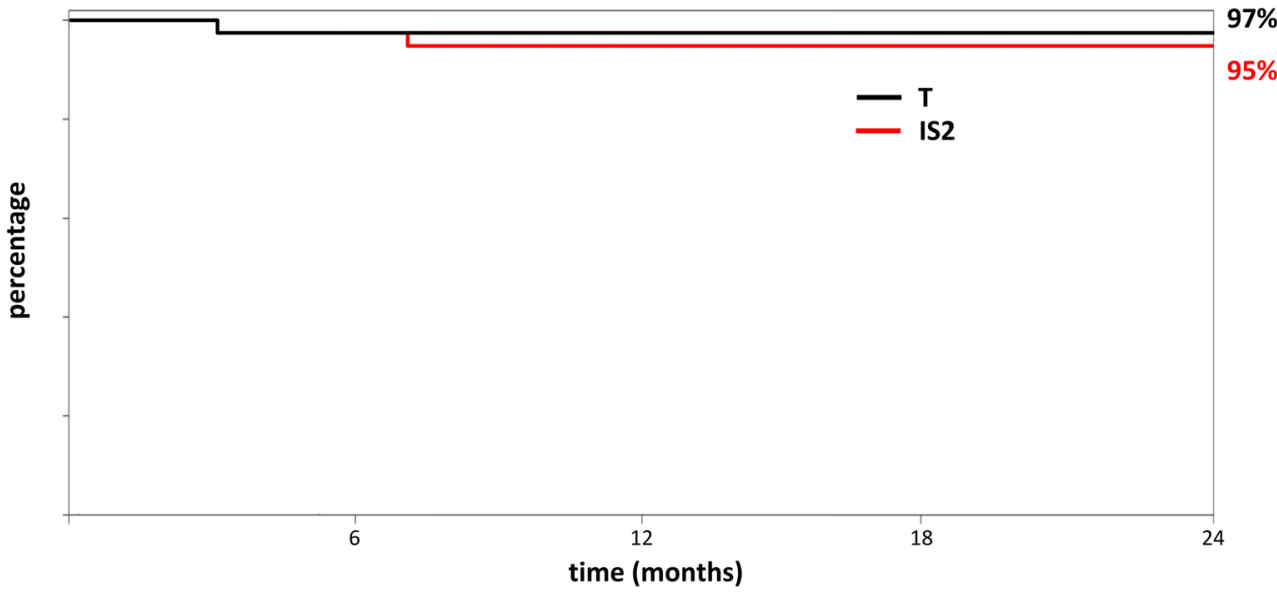


easily be discovered by other analysis methods. Because of this, the IOP and medication changes seen here should not readily be generalized to patients who undergo these procedures but have other baseline parameters. For instance, patients with a high preoperative IOP undergoing $\mathrm{T}$ typically have a much more substantial IOP reduction than those with a low preoperative IOP, as examined here [29, 30]. All patients also had phacoemulsification, which can lower IOP on its own [28]. It has been speculated that the IOP reduction after phacoemulsification is caused by a trabeculoplasty-like reaction $[3,38]$. IS2 might have been inadvertently helped by this additional effect [39]. In $\mathrm{T}$ in contrast, there is no significant contribution from cataract surgery to IOP reduction $[40,41]$. Presumably, this is the case because most of the conventional outflow occurs through the 120 to $180^{\circ}$ of nasal, unroofed Schlemm's canal and is unaffected by the remaining trabecular meshwork [42]. However in IS2, the nasal Schlemm's canal remains covered by glaucomatous TM and can be impacted by a trabeculoplasty-like effect of cataract surgery.

In summary, a highly balanced comparison of IS 2 and $\mathrm{T}$ by exact matching showed that reduction of IOP and the number of glaucoma medications is lower and sustained in T. IOP in IS2 rebounds 6 to 24 months after surgery, eventually reaching preoperative levels.

Funding Open Access funding enabled and organized by Projekt DEAL. Dr. Nils Loewen received a departmental grant from the University of Wuerzburg.

\section{Compliance with ethical standards}

Conflict of interest NAL has received a lecture honorarium from Neomedix Corp. All other authors certify that they have no affiliations with or involvement in any organization or entity with any financial interest (such as honoraria; educational grants; participation in speakers' bureaus; membership, employment, consultancies, stock ownership, or other equity interest; and expert testimony or patent-licensing arrangements), or non-financial interest (such as personal or professional relationships, affiliations, knowledge or beliefs) in the subject matter or materials discussed in this manuscript.

Ethical approval All procedures performed in studies involving human participants were in accordance with the ethical standards of the University of Würzburg and with the 1964 Helsinki declaration and its later amendments or comparable ethical standards.

Informed consent This type of study does not require informed consent.

Open Access This article is licensed under a Creative Commons Attribution 4.0 International License, which permits use, sharing, adaptation, distribution and reproduction in any medium or format, as long as you give appropriate credit to the original author(s) and the source, provide a link to the Creative Commons licence, and indicate if changes were made. The images or other third party material in this article are included in the article's Creative Commons licence, unless indicated otherwise in a credit line to the material. If material is not included in the article's Creative Commons licence and your intended use is not permitted by statutory regulation or exceeds the permitted use, you will need to obtain permission directly from the copyright holder. To view a copy of this licence, visit http://creativecommons.org/licenses/by/4.0/.

\section{References}

1. Capitena Young CE, Ammar DA, Seibold LK et al (2018) Histopathologic examination of trabecular meshwork changes after trabecular bypass stent implantation. J Glaucoma 27:606-609

2. Shah M, Campos-Möller X, Werner L et al (2018) Midterm failure of combined phacoemulsification with trabecular microbypass stenting: Clinicopathological analysis. J Cataract Refract Surg 44: 654-657

3. Esfandiari H, Taubenslag K, Shah P et al (2019) Two-year data comparison of ab interno trabeculectomy and trabecular bypass stenting using exact matching. J Cataract Refract Surg 45:608-614

4. Kaplowitz K, Bussel II, Honkanen R et al (2016) Review and metaanalysis of ab-interno trabeculectomy outcomes. Br J Ophthalmol 100:594-600

5. Haubold B, Börsch-Haubold A (2017) Exact matching. In: Haubold B, Börsch-Haubold A (eds) Bioinformatics for evolutionary biologists: a problems approach. Springer International Publishing, Cham, pp 47-67

6. Akil H, Chopra V, Huang A et al (2016) Clinical results of ab interno trabeculotomy using the Trabectome in patients with pigmentary glaucoma compared to primary open angle glaucoma. Clin Experiment Ophthalmol 44:563-569

7. Khan M, Saheb H, Neelakantan A et al (2015) Efficacy and safety of combined cataract surgery with 2 trabecular microbypass stents versus ab interno trabeculotomy. J Cataract Refract Surg 41:1716 1724

8. Kurji K, Rudnisky CJ, Rayat JS et al (2017) Phaco-trabectome versus phaco-iStent in patients with open-angle glaucoma. Can J Ophthalmol 52:99-106

9. Dang Y, Waxman S, Wang C et al (2018) Intraocular pressure elevation precedes a phagocytosis decline in a model of pigmentary glaucoma. F1000Res 7:174

10. Sherwood ME, Richardson TM (1988) Phagocytosis by trabecular meshwork cells: sequence of events in cats and monkeys. Exp Eye Res 46:881-895

11. Streilein JW, Masli S, Takeuchi M, Kezuka T (2002) The eye's view of antigen presentation. Hum Immunol 63:435-443

12. Stein-Streilein J, Streilein JW (2002) Anterior chamber associated immune deviation (ACAID): regulation, biological relevance, and implications for therapy. Int Rev Immunol 21:123-152

13. Bussel II, Kaplowitz K, Schuman JS et al (2015) Outcomes of ab interno trabeculectomy with the trabectome by degree of angle opening. Br J Ophthalmol 99:914-919

14. Bussel II, Kaplowitz K, Schuman JS et al (2014) Outcomes of ab interno trabeculectomy with the trabectome after failed trabeculectomy. Br J Ophthalmol 99:258-262

15. King G Nearest neighbor matching. https://r.iq.harvard.edu/docs/ matchit/2.4-15/Nearest_Neighbor_Match.html. Accessed 8 Jun 2018

16. Core Team R (2018) R: A language and environment for statistical computing. R Foundation for Statistical Computing, Vienna

17. Samuelson TW, Sarkisian SR Jr, Lubeck DM et al (2019) Prospective, randomized, controlled pivotal trial of an ab interno implanted trabecular micro-bypass in primary open-angle glaucoma and cataract: two-year results. Ophthalmology 126:811-821

18. Fallano K, Bussel I, Kagemann L et al (2017) Training strategies and outcomes of $a b$ interno trabeculectomy with the trabectome. F1000Res 6:67 
19. Francis BA, Minckler D, Dustin L et al (2008) Combined cataract extraction and trabeculotomy by the internal approach for coexisting cataract and open-angle glaucoma: initial results. J Cataract Refract Surg 34:1096-1103

20. Kaplowitz K, Loewen NA (2014) Minimally Invasive glaucoma surgery: trabeculectomy ab interno. In: Samples JR, Ahmed IIK (eds) Surgical innovations in glaucoma. Springer, New York, pp 175-186

21. Kaplowitz K, Bussel I, Loewen NA (2015) Trabeculectomy by internal approach: Trabectome. In: Francis B, Sarkisian S, Tan J (eds) Minimally Invasive Glaucoma Surgery: the Science and the Practice. Thieme, pp 82-91

22. Toth M, Bazeer S, Gazzard G (2016) Retrospective review of pressure reducing effect of iStent and Trabectome procedures combined with cataract surgery. Acta Ophthalmol 94. https://doi.org/10.1111/ j.1755-3768.2016.0466

23. Blackwell M, Iacus S, King G, Porro G (2009) Cem: coarsened exact matching in Stata. Stata J 9:524-546

24. King G Exact Matching. https://r.iq.harvard.edu/docs/matchit/2.415/Exact_Matching.html. Accessed 9 Jul 2018

25. Burden A, Roche N, Miglio C et al (2017) An evaluation of exact matching and propensity score methods as applied in a comparative effectiveness study of inhaled corticosteroids in asthma. Pragmat Obs Res 8:15-30

26. Guedes RAP, Gravina DM, Lake JC et al (2019) Intermediate results of iStent or iStent inject implantation combined with cataract surgery in a real-world setting: a longitudinal retrospective study. Ophthalmol Ther 8:87-100

27. Minckler DS, Baerveldt G, Alfaro MR, Francis BA (2005) Clinical results with the Trabectome for treatment of open-angle glaucoma. Ophthalmology 112:962-967

28. Samuelson TW, Katz LJ, Wells JM et al (2011) Randomized evaluation of the trabecular micro-bypass stent with phacoemulsification in patients with glaucoma and cataract. Ophthalmology 118:459-467

29. Loewen RT, Roy P, Parikh HA et al (2016) Impact of a glaucoma severity index on results of trabectome surgery: larger pressure reduction in more severe glaucoma. PLoS One 11:e0151926

30. Roy P, Loewen RT, Dang Y et al (2017) Stratification of phacotrabectome surgery results using a glaucoma severity index in a retrospective analysis. BMC Ophthalmol 17:30

31. Mosaed S, Chak G, Haider A et al (2015) Results of trabectome surgery following failed glaucoma tube shunt implantation: cohort study. Medicine 94:e1045
32. Dang Y, Wang C, Shah P et al (2018) Outflow enhancement by three different $a b$ interno trabeculectomy procedures in a porcine anterior segment model. Graefes Arch Clin Exp Ophthalmol 256: $1305-1312$

33. Abbas A, Agrawal P, King AJ (2018) Exploring literature-based definitions of hypotony following glaucoma filtration surgery and the impact on clinical outcomes. Acta Ophthalmol 96:e285-e289

34. Sii S, Triolo G, Barton K (2019) Case series of hypotony maculopathy after CyPass insertion treated with intra-luminal suture occlusion. Clin Experiment Ophthalmol 47:679-680

35. Tan SZ, Walkden A, Au L (2018) One-year result of XEN45 implant for glaucoma: efficacy, safety, and postoperative management. Eye 32:324-332

36. McDonnell F, Dismuke WM, Overby DR, Stamer WD (2018) Pharmacological regulation of outflow resistance distal to Schlemm's canal. Am J Physiol Cell Physiol 315:C44-C51

37. Waxman S, Wang C, Dang Y et al (2018) Structure-function changes of the porcine distal outflow tract in response to nitric oxide. Invest Ophthalmol Vis Sci 59:4886-4895

38. Wang N, Chintala SK, Fini ME, Schuman JS (2003) Ultrasound activates the TM ELAM-1/IL-1/NF-kappaB response: a potential mechanism for intraocular pressure reduction after phacoemulsification. Invest Ophthalmol Vis Sci 44:1977-1981

39. Malvankar-Mehta MS, Iordanous Y, Chen YN et al (2015) iStent with phacoemulsification versus phacoemulsification alone for patients with glaucoma and cataract: a meta-analysis. PLoS One 10: e0131770

40. Parikh HA, Bussel II, Schuman JS et al (2016) Coarsened exact matching of phaco-trabectome to trabectome in phakic patients: lack of additional pressure reduction from phacoemulsification. PLoS One 11:e0149384

41. Neiweem AE, Bussel II, Schuman JS et al (2016) Glaucoma surgery calculator: limited additive effect of phacoemulsification on intraocular pressure in ab interno trabeculectomy. PLoS One 11: $\mathrm{e} 0153585$

42. Parikh HA, Loewen RT, Roy P et al (2016) Differential canalograms detect outflow changes from trabecular microbypass stents and ab interno trabeculectomy. Sci Rep 6:34705

Publisher's note Springer Nature remains neutral with regard to jurisdictional claims in published maps and institutional affiliations. 